\title{
Sympathetic overactivity in active ulcerative colitis: effects of clonidine
}

\author{
Raffaello Furlan, ${ }^{1}$ Sandro Ardizzone,${ }^{2}$ Laura Palazzolo, ${ }^{1}$ Alexandra Rimoldi, ${ }^{1}$ Francesca Perego, ${ }^{1}$ \\ Franca Barbic, ${ }^{1}$ Maurizio Bevilacqua, ${ }^{3}$ Luca Vago, ${ }^{4}$ Gabriele Bianchi Porro, ${ }^{2}$ and Alberto Malliani ${ }^{1}$ \\ ${ }^{1}$ Unità Sincopi, Medicina Interna II; and ${ }^{2}$ Gastroenterologia, ${ }^{3}$ Endocrinologia, and ${ }^{4}$ Anatomia Patologica, \\ Ospedale L. Sacco, Dipartimento Scienze Cliniche L. Sacco, Università degli Studi di Milano, Milan, Italy
}

Submitted 22 June 2005; accepted in final form 24 August 2005

\begin{abstract}
Furlan, Raffaello, Sandro Ardizzone, Laura Palazzolo, Alexandra Rimoldi, Francesca Perego, Franca Barbic, Maurizio Bevilacqua, Luca Vago, Gabriele Bianchi Porro, and Alberto Malliani. Sympathetic overactivity in active ulcerative colitis: effects of clonidine. Am J Physiol Regul Integr Comp Physiol 290: R224-R232, 2006. First published August 25, 2005; doi:10.1152/ajpregu.00442.2005.-Previous reports suggest that inflammatory bowel diseases may be accompanied by abnormalities in the neural autonomic profile. We tested the hypotheses that 1) an exaggerated sympathetic activity characterizes active ulcerative colitis (UC) and 2) a reduction of sympathetic activity by clonidine would be associated with clinical changes of UC. In 23 patients with UC and 20 controls, muscle sympathetic nerve activity (MSNA), ECG, blood pressure, and respiration were continuously recorded, and plasma catecholamine was evaluated both at rest and during a $75^{\circ}$ head-up tilt. Autonomic profile was assessed by MSNA, norepinephrine, epinephrine, spectral markers of low-frequency (LF) cardiac sympathetic ( $\mathrm{LF}_{\mathrm{RR}}$; normalized units) and highfrequency $(\mathrm{HF})$ parasympathetic $\left(\mathrm{HF}_{\mathrm{RR}}\right.$; normalized units) modulation and sympathetic vasomotor control (LF systolic arterial pressure; $\mathrm{LF}_{\mathrm{SAP}}$ ), obtained by spectrum analysis of the R-R interval and systolic pressure variability. Among UC patients, 16 agreed to be randomly assigned to 8 -wk transdermal clonidine ( $15 \mathrm{mg} / \mathrm{wk}, 9$ subjects), or placebo (7 patients). An autonomic profile, Disease Activity Index (DAI), and endoscopic pattern were compared before and after clonidine/placebo. At rest, MSNA, heart rate (HR), $\mathrm{LF}_{\mathrm{RR}}, \mathrm{LF} / \mathrm{HF}$, and $\mathrm{LF}_{\mathrm{SAP}}$ were higher and $\mathrm{HF}_{\mathrm{RR}}$ was lower in patients than in controls. Tilt decreased $\mathrm{HF}_{\mathrm{RR}}$ and increased MSNA and $L F_{\mathrm{RR}}$ less in patients than in controls. Clonidine decreased HR, MSNA, epinephrine, $\mathrm{LF}_{\mathrm{RR}}$, and increased $\mathrm{HF}_{\mathrm{RR}}$, whereas placebo had no effects. Changes of the autonomic profile after clonidine were associated with reduction of DAI score. An overall increase of sympathetic activity characterized active UC. Normalization of the autonomic profile by clonidine was accompanied by an improvement of the disease.
\end{abstract}

sympathetic nervous system; microneurography; power spectrum analysis; tilt test.

IMPORTANCE OF THE AUTONOMIC nervous system in modulating motility, secretion, and mucosal immunity of the gastrointestinal tract is widely recognized $(4,6,12,39,43)$. There is increasing evidence that autonomic abnormalities may play a role also in the pathophysiology of the gastrointestinal apparatus, namely in inflammatory disorders.

In animals, alterations of the sympathetic nervous system activity were found to facilitate and sustain local inflammatory mechanisms in ulcerative colitis (UC) after the onset of mucosal lesions (8). Accordingly, in rats with experimental colitis, there was a reduced degree of colonic tissue damage if they had

Address for reprint requests and other correspondence: R. Furlan, Unità Sincopi e Disturbi della Postura, Medicina Interna II, Ospedale L. Sacco, Università di Milano, Via G.B. Grassi 74, 20157 Milano, Italy (e-mail: raffaello.furlan@unimi.it). undergone a previous sympathectomy compared with animals with intact autonomic nervous system (26).

In humans, the attempt to identify whether autonomic alterations are likely to be present in UC has furnished inconclusive results. The promoting role of an increased overall sympathetic activity in sustaining the inflammatory process of UC may be inferred from the fact that psychological stress can reactivate quiescent UC (42). On the other hand, the increase of parasympathetic drive to the organs by nicotine patch was found to ameliorate the inflammatory state in UC patients (32). However, these human studies did not address the relationship between autonomic abnormalities and the activity of the disease. These aspects may be of paramount importance because the possibility of manipulating an abnormal autonomic profile by drugs might result in an amelioration of the disease.

In the present study, we compared the cardiovascular autonomic control of patients with mild, active UC and healthy controls both at rest and during a tilt maneuver, a stimulus that promotes an overall increase of the sympathetic activity. The autonomic profile was assessed by means of the direct recording of postganglionic muscle sympathetic nerve activity (MSNA) and the evaluation of plasma norepinephrine and epinephrine levels. Spectral markers of cardiac sympathetic and vagal modulation of the sinoatrial node activity and of vascular sympathetic regulation, obtained by power spectrum analysis of heart period and systolic arterial pressure (SAF) variability, completed the autonomic assessment of each patient. As a second aim, we tested the hypothesis that, in a state of sympathetic overactivity, the neural changes induced by clonidine administration would be associated with an improvement of the disease.

\section{MATERIALS AND METHODS}

\section{Study Design and Population}

The present investigation includes two separate parts, as outlined in Fig. 1. Twenty-three patients with active UC (age, $42 \pm 3$ yr; 15 men, 8 women) were compared with 20 healthy controls (age, $38 \pm 3 \mathrm{yr}$; 12 men, 8 women), both at rest and during a $75^{\circ}$ head-up tilt.

Among the group of patients with active UC, a subgroup of 16 patients (age, $44 \pm 4 \mathrm{yr} ; 12$ men, 4 women) agreed to undergo a second part of the protocol and was randomly assigned to 8-wk of transdermal clonidine (Catapresan TTS 2; Boehringer-Ingelheim; 15 $\mathrm{mg} / \mathrm{wk} ; n=9,6 \mathrm{men}, 3$ women; age, $44 \pm 6 \mathrm{yr})$ or placebo patch $(n=$ 7; 6 men, 1 female; age, $47 \pm 6 \mathrm{yr}$ ). All of the subjects attending the second part of the protocol were studied only in the recumbent position. Their previous therapy, if any, was maintained unchanged for the $8 \mathrm{wk}$ of the study (Table 1) and clonidine or placebo patches

\footnotetext{
The costs of publication of this article were defrayed in part by the payment of page charges. The article must therefore be hereby marked "advertisement" in accordance with 18 U.S.C. Section 1734 solely to indicate this fact.
} 


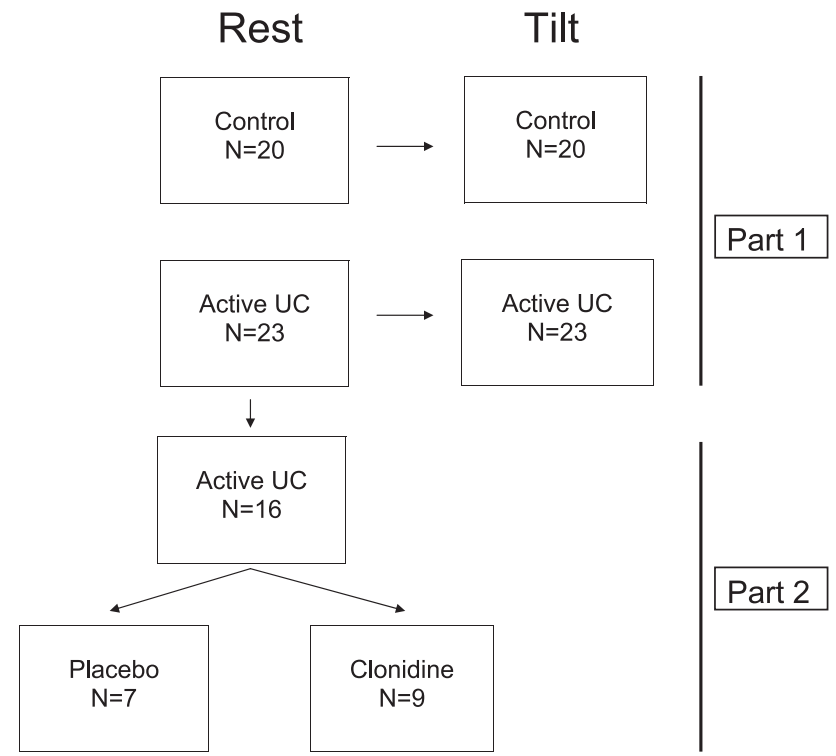

Fig. 1. Flow diagram of the study protocol. UC, ulcerative colitis.

were added. Patches were placed on arms or trunk and changed every 7 days. Two patients had not received any drug before the study because the diagnosis of UC was made just before their enrollment (Table 1).

Control subjects were healthy volunteers without evidence of organic disease on the basis of the interview and physical examination. Subjects with UC were part of a population attending the outpatient clinic for inflammatory bowel disease of the gastroenterology unit of the hospital, who agreed to participate in the study. UC was diagnosed on the basis of clinical and radiographic signs, endos-

Table 1. Demographic and clinical features of patients with UC

\begin{tabular}{|c|c|}
\hline & UC Patients \\
\hline Total number & 23 \\
\hline \multicolumn{2}{|l|}{ Gender } \\
\hline Male & $15(65)$ \\
\hline Female & $8(35)$ \\
\hline \multicolumn{2}{|l|}{ Age, yr } \\
\hline Mean $\pm \mathrm{SD}$ & $42 \pm 3$ \\
\hline \multicolumn{2}{|l|}{ Weight, kg } \\
\hline Mean \pm SD & $68 \pm 10$ \\
\hline \multicolumn{2}{|l|}{ Height, cm } \\
\hline Mean \pm SD & $171 \pm 56$ \\
\hline \multicolumn{2}{|l|}{ Smoking status } \\
\hline Smoker & $9(39)$ \\
\hline Former smoker or nonsmoker & $14(61)$ \\
\hline \multicolumn{2}{|l|}{ Duration of disease, $\mathrm{yr}$} \\
\hline Mean \pm SD & $5.6 \pm 1.3$ \\
\hline \multicolumn{2}{|l|}{ Extent of disease } \\
\hline Proctosigmoiditis & $11(48)$ \\
\hline Left-sided colitis & $9(39)$ \\
\hline Pancolitis & $3(13)$ \\
\hline \multicolumn{2}{|l|}{ Therapy } \\
\hline No drug & $2(9)$ \\
\hline Systemic steroids (prednisone $25-50 \mathrm{mg} /$ day) & $4(17)$ \\
\hline Topical steroids (beclomethasone $3 \mathrm{mg} /$ day) & $7(30)$ \\
\hline Mesalamine or sulfasalazine $(1.2-2.0 \mathrm{~g} /$ day $)$ & $21(91)$ \\
\hline Topical mesalamine (2-4 g/day) & $16(70)$ \\
\hline Cyclosporin $\left(4 \mathrm{mg} \cdot \mathrm{kg}^{-1} \cdot \mathrm{day}^{-1}\right)$ & $1(4)$ \\
\hline
\end{tabular}

Values are number of patients; values in parentheses are percentages. UC, ulcerative colitis. copy, and pathology criteria. Demographic and clinical features of the group of patients with UC are reported in Table 1.

\section{Inclusion Criteria}

Subjects older than $18 \mathrm{yr}$ with diagnosed UC were eligible to be enrolled, if they were suffering from clinically and endoscopically active disease. UC was defined "active" on the basis of a Disease Activity Index (DAI) score $>4$ (see below).

\section{Exclusion Criteria}

Patients with UC were excluded if hepatic, renal, lung, heart diseases, infective colitis, secondary causes of autonomic dysfunction, and pregnancy were present, on the basis of the history, complete medical evaluation, and routine tests. No subject was enrolled if contraindications to the use of clonidine were present.

\section{Disease Activity Assessment}

Activity of UC was quantified by a DAI (3) that measured stool frequency, rectal bleeding, endoscopic findings, and the physician's evaluation of the disease severity by a $0-3$ gravity scale (35). The highest score, i.e., 12, indicates more than five evacuations than subject's normal, blood alone passed, spontaneous bleeding with ulcerations at the endoscopic evaluation, and severe disease as globally assessed by the physician. Conversely, a score of 0 indicates normal stool frequency, no rectal bleeding, normal mucosal appearance during endoscopy, and no clinical signs of disease activity. Details about parameters, classification, and point scales of DAI are provided in Table 2 .

Truelove and Richards' $0-4$ scale (40) was used to assess the degree of colonic inflammatory lesions on biopsies obtained during each of the colonoscopies performed at the time of the first study and after $8 \mathrm{wk}$ of clonidine/placebo treatment.

\section{Recorded Variables}

In every subject, ECG, arterial pressure (Finapres device; Ohmeda, Louisville, CO), respiratory activity (thoracic bellows), and MSNA were continuously recorded.

MSNA was obtained by a microneurography technique (41). Briefly, a unipolar tungsten electrode was placed in the right peroneal nerve near the fibular head for multiunit postganglionic sympathetic nerve recording. A reference electrode was inserted subcutaneously and close to the recording needle. The row neural signal was amplified

Table 2. Parameters, classification and point scales of the Disease Activity Index modified from Ref. 3

\begin{tabular}{llr}
\hline \hline \multicolumn{1}{c}{ Parameter } & \multicolumn{1}{c}{ Classification } & $\begin{array}{r}\text { Point } \\
\text { Scales }\end{array}$ \\
\hline Clinical, stool frequency (on & Normal & 0 \\
a weekly basis) & $1-2$ evacuations/day > normal & 1 \\
& $3-4$ evacuations/day > normal & 2 \\
& $>4$ evacuations/day $>$ normal & 3 \\
Clinical, rectal bleeding & None & 0 \\
& Streaks of blood & 1 \\
& Evident blood & 2 \\
Endoscopy, mucosal & Mostly blood & 3 \\
appearance & Normal & 0 \\
& Mild friability & 1 \\
Clinical, physician's rating of & Moderate friability & 2 \\
disease activity & Exudation, spontaneous bleeding & 3 \\
& Normal & 0 \\
Maximum score & Mild & 1 \\
& Moderate & 2 \\
& Severe & 3
\end{tabular}


(1,000-fold amplification), fed to a band-pass filter (bandwidth between 700 and 2,000 Hz), rectified, and integrated (time constant = $0.1 \mathrm{~s}$ ) by a nerve traffic analyzer system (Bioengineering Dept., University of Iowa, Iowa City, IA). Systemic arterial pressure, ECG, integrated MSNA, and the respiratory signal were digitized at 300 samples/s and stored on the hard disk of a personal computer for offline analysis. Plasma epinephrine and norepinephrine were measured on venous blood samples by high-performance liquid chromatography with electrochemical detection.

Every patient underwent a colonoscopy when enrolled in the study. A second colonoscopy was performed at the end of the 8-wk placebo/ clonidine study only in those patients who agreed to participate in the second part of the protocol.

\section{Protocol}

The experimental protocol was approved by the Hospital Review Board in Human Research, and written informed consent was provided by all subjects.

Part 1. After instrumentation and an adequate period of adaptation on a tilt table, ECG, beat-by-beat arterial pressure, respiratory activity, and MSNA were continuously recorded for $15 \mathrm{~min}$ in the recumbent position, and a blood sample was obtained for evaluation of plasma catecholamine. Thereafter, all subjects were tilted at $15^{\circ}$ intervals every 4 min until the $75^{\circ}$ head-up position was reached. This position was maintained for $15 \mathrm{~min}$. At minute 5 of the $75^{\circ}$ tilt, a second blood sample was withdrawn.

Part 2. After having completed part 1 of the protocol, patients with UC were asked to participate also in part 2 of the study that was illustrated in detail. The 16 subjects who agreed to take part were randomly assigned to an 8-wk placebo or a clonidine transdermal patch. After each period, these patients underwent a second 15-min data recording, including the direct evaluation of the MSNA and plasma catecholamine assessment in the recumbent position. In addition, they were clinically and endoscopically reevaluated by both the DAI scale and a colonoscopy at the end of the 8-wk period of placebo or clonidine assumption. We were unable, for ethical reasons, to perform a crossover between the placebo and clonidine groups because of the need of a third peroneal nerve puncture for MSNA recording and colonoscopy in each patient.

\section{Data Analysis}

Microneurography recordings were considered to reflect MSNA, according to criteria previously defined (41). The principles of the software for data acquisition and autoregressive spectral and crossspectral analysis have been described elsewhere $(5,29)$. Briefly, two major oscillatory components can be extracted from R-R interval spontaneous variability (38). A high-frequency $\left(\mathrm{HF}_{\mathrm{RR}}, 0.25 \mathrm{~Hz}\right)$ component, the power of which is considered a marker of the vagal modulation of the sinoatrial node discharge (1), and a low-frequency $\left(\mathrm{LF}_{\mathrm{RR}}, 0.10 \mathrm{~Hz}\right)$ component considered, when normalized, an index of the cardiac sympathetic modulation and of its changes $(14,20,25$, 29). The power of these two different oscillatory components is computed both in absolute $\left(\mathrm{ms}^{2}\right)$ and normalized units. Normalized units are obtained by dividing the absolute power of each spectral component by total power minus the very low-frequency (LF) component $(<0.03 \mathrm{~Hz})$ and multiplying by $100(25,29)$. The LF-to-HF ratio quantifies the reciprocal changes of the sympathetic and vagal modulation of the sinoatrial node discharge $(25,29) . \operatorname{LF}_{\mathrm{SAP}}(0.10 \mathrm{~Hz})$ can be considered a marker of the sympathetic modulation of vasomotor activity $(14,25,29,30)$.

\section{Statistics}

Data are expressed as means \pm SE. One-way ANOVA was used to assess the differences between patients and controls at rest and to compare the changes induced by the tilt maneuver between the two groups.
Modifications induced by clonidine or placebo in respect to the active phase of the disease were evaluated by Student's $t$-test for paired observations. Differences were considered significant at values of $P<0.05$.

\section{RESULTS}

\section{Autonomic Profile of UC at Rest}

Heart rate was significantly higher in subjects with UC than in controls, whereas blood pressure and respiratory activity were similar in the two groups (Table 3). Neural sympathetic discharge (MSNA) was higher in patients suffering from UC than in controls (Fig. 2 and Table 3). Plasma norepinephrine and epinephrine levels were only slightly greater in patients (Table 3). Figure 3 illustrates examples of power spectrum analysis of R-R interval and SAP variability and of respiration in a control subject and in a patient with active UC. Notice that in the patient, the low-frequency components of both R-R interval and SAP variability were higher than in the control subject. Accordingly, $\mathrm{LF}_{\mathrm{RR}}$ normalized units and $\mathrm{LF}_{\mathrm{SAP}}$ were significantly higher in the group of patients than in controls, whereas the marker of vagal modulation of the sinoatrial node activity, $\mathrm{HF}_{\mathrm{RR}}$, showed an opposite pattern (Table 3). Finally, the LF-to-HF ratio was greater in UC patients, suggesting a shift of the cardiac sympathovagal balance toward a sympathetic predominance, compared with controls (Table 3).

\section{Changes Induced by $75^{\circ}$ Head-Up Tilt}

The gravitational stimulus induced similar changes in heart rate, blood pressure, respiration, plasma norepinephrine, and epinephrine in patients with UC and in healthy controls (Table 4). The increase of MSNA in response to tilt was larger in controls than in UC patients. Figure 4 shows the power spectra of R-R interval and SAP variability and of respiration during tilt in the same control subject and patient of Fig. 3. Notice that the spectral profiles were similar in both individuals. Thus

Table 3. Hemodynamic and respiratory parameters and indices of autonomic activity in controls and patients with active $U C$ in recumbent position

\begin{tabular}{|c|c|c|}
\hline & Controls & $\mathrm{UC}$ \\
\hline $\mathrm{SAP}, \mathrm{mmHg}$ & $122 \pm 3$ & $120 \pm 2$ \\
\hline $\mathrm{DAP}, \mathrm{mmHg}$ & $74 \pm 2$ & $76 \pm 2$ \\
\hline $\mathrm{HR}$, beats/min & $63 \pm 2$ & $75 \pm 30 * *$ \\
\hline Resp, cycles/min & $17 \pm 1$ & $18 \pm 1$ \\
\hline MSNA, bursts/min & $16 \pm 2.1$ & $33 \pm 3.5 * *$ \\
\hline bursts/100 beats & $28 \pm 4$ & $56 \pm 6.3 * *$ \\
\hline $\mathrm{NE}, \mathrm{pg} / \mathrm{ml}$ & $266 \pm 31$ & $316 \pm 39$ \\
\hline $\mathrm{E}, \mathrm{pg} / \mathrm{ml}$ & $35 \pm 6.0$ & $44 \pm 6.2$ \\
\hline $\mathrm{RR}$ variance, $\mathrm{ms}^{2}$ & $2,607 \pm 597$ & $1,405 \pm 290$ \\
\hline $\mathrm{LF}_{\mathrm{RR}}, \mathrm{ms}^{2}$ & $734 \pm 183$ & $701 \pm 185$ \\
\hline nu & $52 \pm 4$ & $74 \pm 2 * *$ \\
\hline $\mathrm{HF}_{\mathrm{RR}}, \mathrm{ms}^{2}$ & $788 \pm 209$ & $188 \pm 47 * *$ \\
\hline nu & $43 \pm 4$ & $20 \pm 1.8 * *$ \\
\hline $\mathrm{LF} / \mathrm{HF}$ & $1.5 \pm 0.2$ & $5.1 \pm 0.9 * *$ \\
\hline $\mathrm{LF}_{\mathrm{SAP}}, \mathrm{mmHg}^{2}$ & $2.2 \pm 0.5$ & $4.1 \pm 0.6^{*}$ \\
\hline
\end{tabular}

SAP, systolic arterial pressure; DAP, diastolic arterial pressure; HR, heart rate; Resp, respiratory activity; MSNA, muscle sympathetic nerve activity; NE, norepinephrine; E, epinephrine; RR, R-R interval; $\mathrm{LF}_{\mathrm{RR}}$, low frequency component of $\mathrm{RR}$ variability; $\mathrm{HF}_{\mathrm{RR}}$, high frequency component; nu, normalized units; $\mathrm{LF}_{\mathrm{SAP}}$, low frequency component of SAP. ${ }^{*} P<0.05$ active UC vs. controls; $* * P<0.01$ active UC vs. controls. 

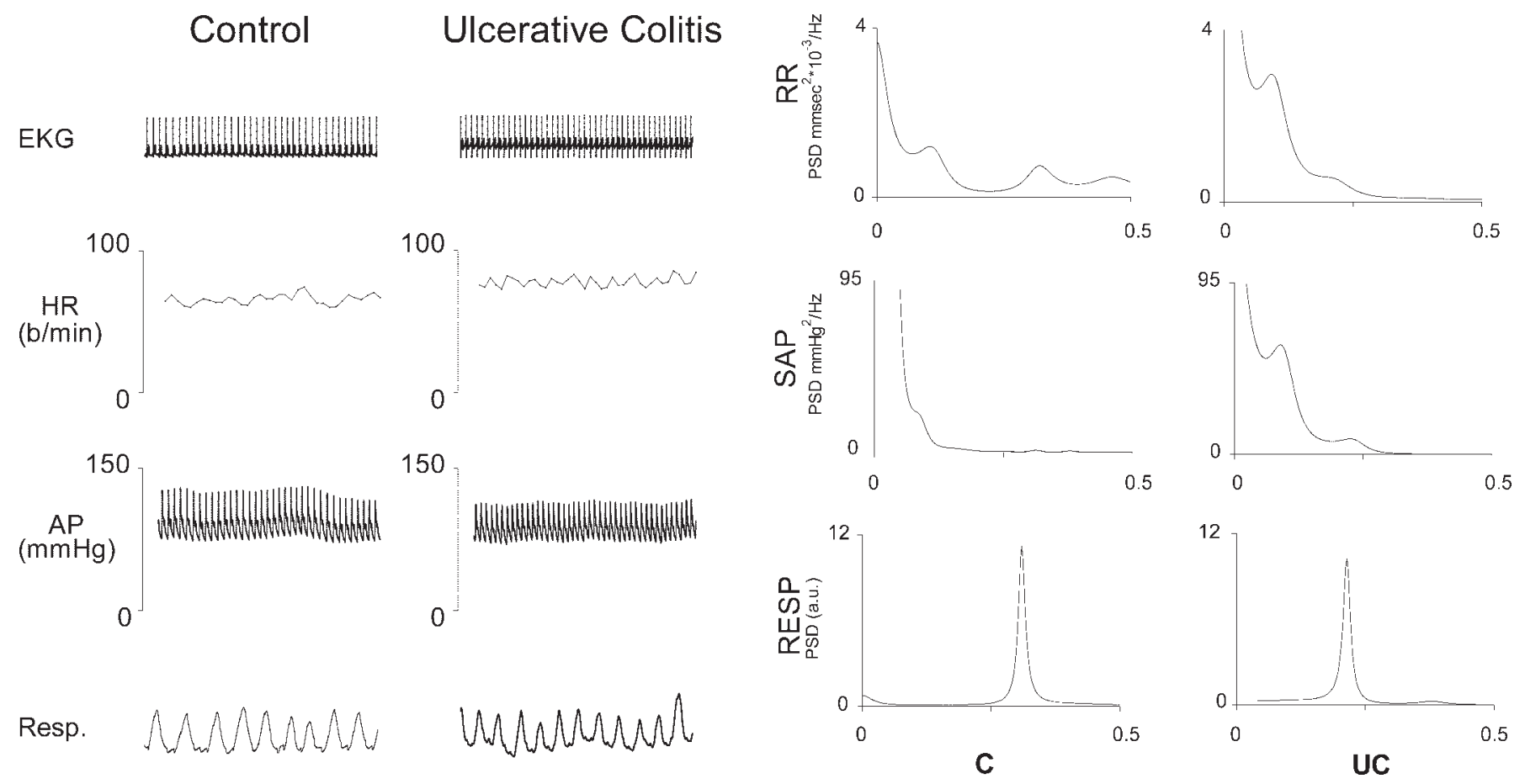

Fig. 3. Examples of power spectrum analyses of R-R interval (RR) and systolic arterial pressure (SAP) variability and Resp in a control subject (C) and patient with active UC (UC) at rest. Notice that in the control subject, the power of the low $\left(\mathrm{LF}_{\mathrm{RR}}\right)$ - and high-frequency $\left(\mathrm{HF}_{\mathrm{RR}}\right)$ oscillations of $\mathrm{R}-\mathrm{R}$ interval variability were similar, suggesting a balance between the sympathetic and vagal cardiac modulations. In contrast, in the patient with $\mathrm{UC}, \mathrm{LF}_{\mathrm{RR}}$ was markedly predominant, indicating a reduced vagal and enhanced sympathetic activity to the sinoatrial node.

Fig. 2. Examples of the recorded variables in a control healthy subject and in a patient with active UC. Notice that heart rate was slightly higher in the patient with UC who showed a more pronounced muscle sympathetic nerve discharge activity (MSNA) than the control. HR, heart rate; AP, arterial pressure; and Resp, respiratory activity.

during tilt, the enhancement of $\mathrm{LF}_{\mathrm{RR}}$ normalized units and $\mathrm{LF} / \mathrm{HF}$ were higher in controls than in UC, and the reduction of $\mathrm{HF}_{\mathrm{RR}}$ was more pronounced in controls than in UC subjects (Table 4).

\section{Autonomic and Clinical Changes After Clonidine}

Placebo did not elicit any change in the hemodynamics, in the indices of autonomic neural control, or in the clinical state of patients with active disease (Table 5 and Fig. 5). Conversely, clonidine administration decreased MSNA (Fig. 5, bottom), plasma epinephrine levels, and the spectral indices of cardiac $\left(\mathrm{LF}_{\mathrm{RR}}\right.$ normalized units) and vascular $\left(\mathrm{LF}_{\mathrm{SAP}}\right)$ sympathetic activity (Table 6). Accordingly, the LF-to-HF ratio decreased, and the marker of cardiac vagal modulation $\mathrm{HF}_{\mathrm{RR}}$ increased (Table 6).

From the clinical standpoint, the reduction of global neural sympathetic activity and increase of vagal cardiac modulation was associated with an improvement of the disease, as globally assessed by the DAI scale (Fig. 6). This was achieved by a concomitant amelioration of symptoms (clinical) and of the endoscopic pattern. Conversely, the colonic histological abnormalities (pathology), observed when the disease was active, did not change significantly after $8 \mathrm{wk}$ of clonidine (Fig. 6, bottom).

\section{DISCUSSION}

Main findings of the present study suggest that, in the recumbent position, patients with active UC were characterized by an overall enhancement of sympathetic activity compared with healthy controls. In addition, patients exhibited a reduced capability to decrease the marker of cardiac vagal drive and to increase the indices of cardiac and vascular sympathetic modulation in response to the gravitational stimulus. Eight-week

Table 4. Changes induced by the $75^{\circ}$ head-up tilt in the hemodynamic and respiratory parameters, and indices of autonomic activity in controls and UC subjects

\begin{tabular}{|c|c|c|}
\hline & Controls & UC \\
\hline $\mathrm{SAP}, \mathrm{mmHg}$ & $1 \pm 2$ & $2 \pm 4$ \\
\hline $\mathrm{DAP}, \mathrm{mmHg}$ & $4 \pm 2$ & $7 \pm 2$ \\
\hline $\mathrm{HR}$, beats/min & $22 \pm 2$ & $17 \pm 2$ \\
\hline Resp, cycles/min & $1 \pm 1$ & $1 \pm 1$ \\
\hline MSNA, bursts/min & $16 \pm 2.2$ & $7.6 \pm 2.3 * *$ \\
\hline bursts/100 beats & $10 \pm 4$ & $-7.5 \pm 5.2$ \\
\hline $\mathrm{NE}, \mathrm{pg} / \mathrm{ml}$ & $192 \pm 38$ & $216 \pm 36$ \\
\hline $\mathrm{E}, \mathrm{pg} / \mathrm{ml}$ & $38 \pm 14$ & $32 \pm 10$ \\
\hline $\mathrm{RR}$ variance, $\mathrm{ms}^{2}$ & $-606 \pm 372$ & $-568 \pm 284$ \\
\hline $\mathrm{LF}_{\mathrm{RR}} \mathrm{ms}^{2}$ & $408 \pm 353$ & $-278 \pm 202$ \\
\hline nu & $29 \pm 5$ & $10.5 \pm 3^{*}$ \\
\hline $\mathrm{HF}_{\mathrm{RR}} \mathrm{ms}^{2}$ & $-662 \pm 189$ & $-142 \pm 44 *$ \\
\hline nu & $-30 \pm 5$ & $-11 \pm 2 * *$ \\
\hline $\mathrm{LF} / \mathrm{HF}$ & $7.9 \pm 1.7$ & $8.9 \pm 2.7$ \\
\hline $\mathrm{LF}_{\mathrm{SAP}}, \mathrm{mmHg}^{2}$ & $9.1 \pm 2.1$ & $9.8 \pm 4.13$ \\
\hline
\end{tabular}



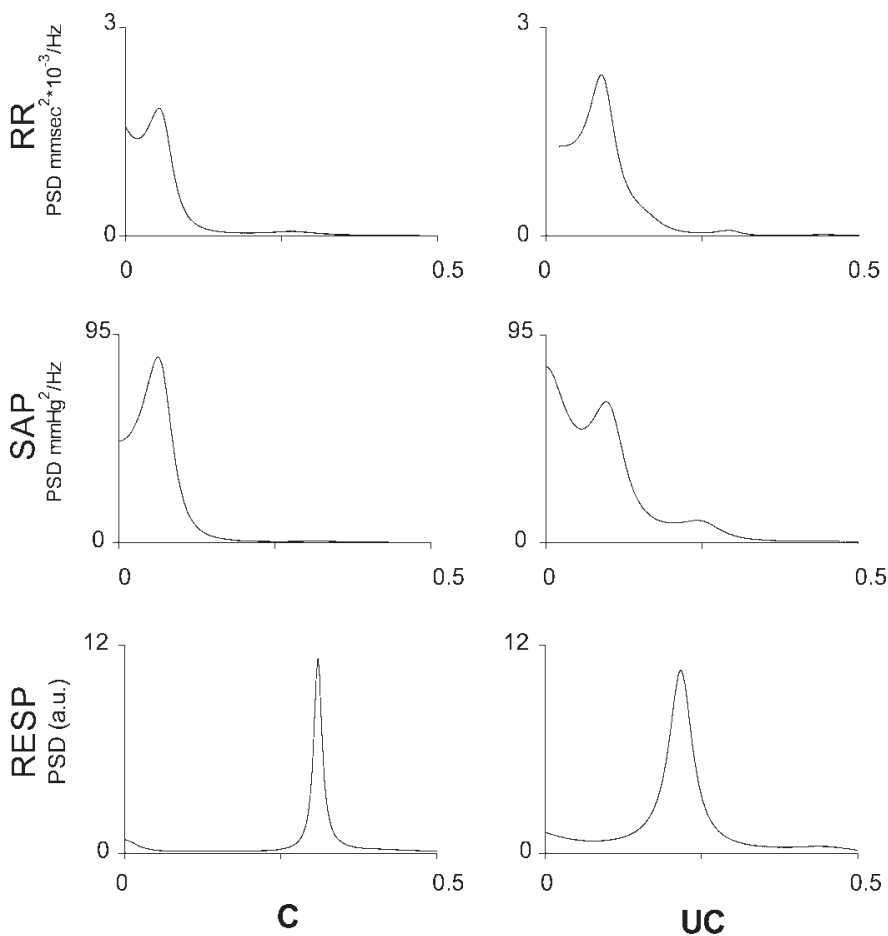

Fig. 4. Same subjects in Fig. 3 are considered during the $75^{\circ}$ head-up tilt. Notice that the power spectra of R-R interval and SAP variability were similar in both individuals. Compared with Fig. 3, the expected increase of LFRR and $\mathrm{LF}_{\mathrm{SAP}}$ was reduced in the patient compared with the control subject.

clonidine administration reduced the sympathetic overactivity observed at rest in patients with active UC. Changes of the autonomic profile induced by clonidine were associated with a clinical and endoscopic improvement.

\section{Autonomic Profile and UC}

Results of the present study suggest that the neural sympathetic discharge activity (MSNA) was markedly higher in subjects with active UC than in healthy controls. In addition, heart rate, $\mathrm{LF}_{\mathrm{RR}}$ normalized units, and LF-to-HF ratio were greater and $\mathrm{HF}_{\mathrm{RR}}$ was lower in patients, suggesting a shift of the sympathovagal modulation of the sinoatrial node toward a sympathetic predominance and parasympathetic reduction, compared with controls. As to this latter aspect, it must be pointed out that power spectrum analysis of R-R interval variability does not provide a direct measure of the neural autonomic activity directed to the heart, but, rather, it furnishes a broad noninvasive marker of the relationship between neural modulation and target organ, i.e., the sinoatrial node responsiveness $(25,29,38)$.

In patients, despite the elevated values of MSNA, plasma norepinephrine was only slightly higher than in controls. It should be recalled that plasma norepinephrine levels provide imprecise estimate of the global neural sympathetic activity. Indeed, only $10-20 \%$ of the norepinephrine released at the level of the sympathetic synapses spills into the circulation or extraneuronal tissues, whereas the large majority undergoes a reuptake process into the sympathetic neuron by the norepinephrine transporter protein $(9,15)$. In addition, plasma norepinephrine levels are also dependent on norepinephrine systemic clearance (9). This latter might have been affected in our study by the likely changes of splanchnic blood flow related to the colonic inflammatory state, similarly to the modifications in blood flow redistribution already described in other diseases (17) during upright posture. Thus changes in norepinephrine transporter protein efficiency and/or in catecholamine systemic clearance, which we could not assess in the present study, might account for the mild increase of plasma norepinephrine in a set of increased sympathetic discharge activity observed in our patients with UC.

Taken together, these findings indicate the existence of an abnormally enhanced sympathetic activity in subjects suffering from active UC.

Because diarrhea characterizes the active phase of UC, the overall increase of sympathetic activity observed in patients of the present study simply could have been related to a contraction of plasma volume. Indeed, unloading of cardiopulmonary and arterial baroreceptor afferents produced by plasma hypovolemia results in a diminished central inhibitory modulation of efferent sympathetic activity, which increases its discharge activity. This leads to vasoconstriction and tachycardia aimed at maintaining blood pressure values. Such hemodynamic and neural changes attending a hypovolemic state are likely to be magnified during the assumption of the upright position, a condition that promotes the additional activation of sympathetic afferents eliciting excitatory positive feedback reflexes from the cardiovascular low-pressure areas (24). In the present study, we did not assess possible plasma volume modifications. However, the gravitational stimulus used, i.e., the tilt maneuver, induced similar increases in heart rate of both patients and controls. In addition, in UC patients, heart rate was stable and did not show the typical slow increase over time observed in conditions characterized by declined plasma volume, such as after weightlessness (27). These findings suggest that the sympathetic overactivity observed in UC at rest is unlikely to be a simple compensatory response to a contraction of plasma volume because it has been also observed in a subset of patients with orthostatic intolerance (17). In addition, if a

Table 5. Hemodynamics respiratory activity, indices of autonomic activity, and hematologic parameters in 7 UC subjects with active disease (symptomatic) and after placebo

\begin{tabular}{|c|c|c|}
\hline & Symptomatic & After Placebo \\
\hline $\mathrm{SAP}, \mathrm{mmHg}$ & $125 \pm 3$ & $126 \pm 5$ \\
\hline $\mathrm{DAP}, \mathrm{mmHg}$ & $81 \pm 4$ & $75 \pm 3$ \\
\hline $\mathrm{HR}$, beats/min & $68 \pm 3$ & $66 \pm 2$ \\
\hline Resp, cycles/min & $17 \pm 2.4$ & $15 \pm 1.8$ \\
\hline MSNA, bursts/min & $40 \pm 4.0$ & $39 \pm 4.5$ \\
\hline bursts/100 beats & $61 \pm 5.9$ & $63 \pm 6.0$ \\
\hline $\mathrm{NE}, \mathrm{pg} / \mathrm{ml}$ & $303 \pm 67$ & $339 \pm 56$ \\
\hline $\mathrm{E}, \mathrm{pg} / \mathrm{ml}$ & $38.5 \pm 13.4$ & $36.5 \pm 17$ \\
\hline $\mathrm{RR}$ variance, $\mathrm{ms}^{2}$ & $1,278 \pm 351$ & $1,614 \pm 350$ \\
\hline $\mathrm{LF}_{\mathrm{RR}} \mathrm{ms}^{2}$ & $499 \pm 94$ & $511 \pm 196$ \\
\hline nu & $71 \pm 4$ & $65 \pm 4$ \\
\hline $\mathrm{HF}_{\mathrm{RR}}, \mathrm{ms}^{2}$ & $158 \pm 56$ & $156 \pm 56$ \\
\hline nu & $20.6 \pm 4$ & $19.8 \pm 4$ \\
\hline $\mathrm{LF} / \mathrm{HF}$ & $5.46 \pm 2.3$ & $3.9 \pm 0.6$ \\
\hline $\mathrm{LF}_{\mathrm{SAP}}, \mathrm{mmHg}^{2}$ & $4.9 \pm 0.6$ & $5.2 \pm 0.8$ \\
\hline $\mathrm{Hb}, \mathrm{g} / \mathrm{dl}$ & $12.9 \pm 0.9$ & $13.3 \pm 0.9$ \\
\hline $\mathrm{RBC}, \times 10^{3} / \mathrm{mm}^{3}$ & $5,115 \pm 295$ & $4,996 \pm 194$ \\
\hline Hct, $\%$ & $39.6 \pm 1.6$ & $40.5 \pm 2.3$ \\
\hline Total protein, g/dl & $7.3 \pm 0.34$ & $7.12 \pm 0.13$ \\
\hline
\end{tabular}

RBC, red blood cells; Hb, hemoglobin; Hct, hematocrit cell pack. 
Symptomatic

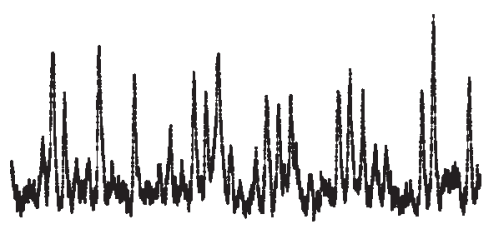

Symptomatic

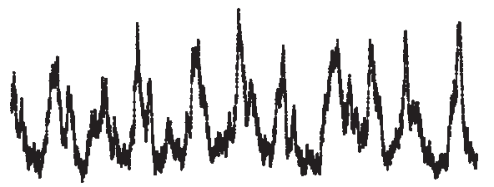

After Placebo

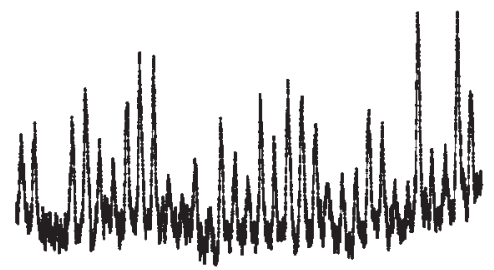

After Clonidine

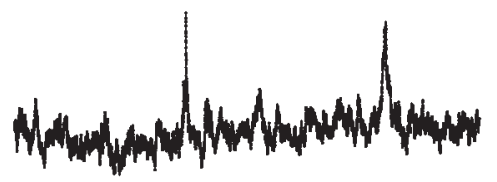

Fig. 5. Neural sympathetic discharge activity of two patients with active UC (left), after placebo, and clonidine. Notice that MSNA was unchanged after placebo administration, whereas it was significantly reduced after clonidine.

$10 \mathrm{sec}$

contracted circulatory volume had been present in patients with $\mathrm{UC}$, the sympathetic response required to adjust to the gravitational stress would have produced a far greater increase of sympathetic efferent traffic. In addition, a larger decrease of cardiac vagal drive would have been evident. Conversely, increase of MSNA and $\mathrm{LF}_{\mathrm{RR}}$ normalized units and decrease of $\mathrm{HF}_{\mathrm{RR}}$ during tilt were less pronounced in subjects with UC than in controls, indicating a reduced capability to adapt to such an excitatory stimulus.

As to the potential mechanisms subserving the sympathetic overactivity in active UC, it can be hypothesized that mediators locally released by the inflamed gut, such as substance P $(2,7$, 19), cytokines (7), and adenosine (2) might induce a chronic stimulation of enteric visceral afferents, resulting in an abnormal afferent impulse traffic to the spinal cord and brain stem. This may induce an increase in the discharge pattern of the

Table 6. Hemodynamics, respiratory activity, indices of autonomic activity, and hematologic parameters in 9 UC subjects with active disease (symptomatic)

and after clonidine

\begin{tabular}{|c|c|c|}
\hline & Symptomatic & After Clonidine \\
\hline $\mathrm{SAP}, \mathrm{mmHg}$ & $120 \pm 4$ & $116 \pm 4$ \\
\hline $\mathrm{DAP}, \mathrm{mmHg}$ & $73 \pm 3$ & $75 \pm 3$ \\
\hline $\mathrm{HR}$, beats/min & $79 \pm 4$ & $77 \pm 4$ \\
\hline Resp, cycles/min & $17 \pm 1.2$ & $16 \pm 1.5$ \\
\hline MSNA, bursts/min & $32 \pm 4.6$ & $21 \pm 4.9^{*}$ \\
\hline bursts/100 beats & $58.5 \pm 8.9$ & $41 \pm 10.3^{*}$ \\
\hline $\mathrm{NE}, \mathrm{pg} / \mathrm{ml}$ & $329 \pm 63$ & $176 \pm 40$ \\
\hline $\mathrm{E}, \mathrm{pg} / \mathrm{ml}$ & $47 \pm 7.6$ & $30 \pm 7.2^{*}$ \\
\hline $\mathrm{RR}$ variance, $\mathrm{ms}^{2}$ & $1,500 \pm 492$ & $3,097 \pm 1393$ \\
\hline $\mathrm{LF}_{\mathrm{RR}}, \mathrm{ms}^{2}$ & $798 \pm 310$ & $879 \pm 518$ \\
\hline $\mathrm{nu}$ & $76 \pm 2.8$ & $47 \pm 6 *$ \\
\hline $\mathrm{HF}_{\mathrm{RR}}, \mathrm{ms}^{2}$ & $200 \pm 71$ & $1,177 \pm 815$ \\
\hline $\mathrm{nu}$ & $21 \pm 3$ & $38 \pm 3 *$ \\
\hline LF/HF & $5.0 \pm 1.1$ & $1.3 \pm 0.2^{*}$ \\
\hline $\mathrm{LF}_{\mathrm{SAP}} \mathrm{mmHg}^{2}$ & $4.5 \pm 0.9$ & $3.0 \pm 0.7$ \\
\hline $\mathrm{Hb}, \mathrm{g} / \mathrm{dl}$ & $14.2 \pm 0.5$ & $14.1 \pm 0.5$ \\
\hline $\mathrm{RBC}, \times 10^{3} / \mathrm{mm}^{3}$ & $4,779 \pm 147$ & $4,737 \pm 177$ \\
\hline Het, \% & $42.7 \pm 1.6$ & $41.9 \pm 1.7$ \\
\hline Total protein, g/dl & $6.97 \pm 0.17$ & $7.01 \pm 0.21$ \\
\hline
\end{tabular}

$* P<0.05$ After Clonidine vs. Symptomatic.
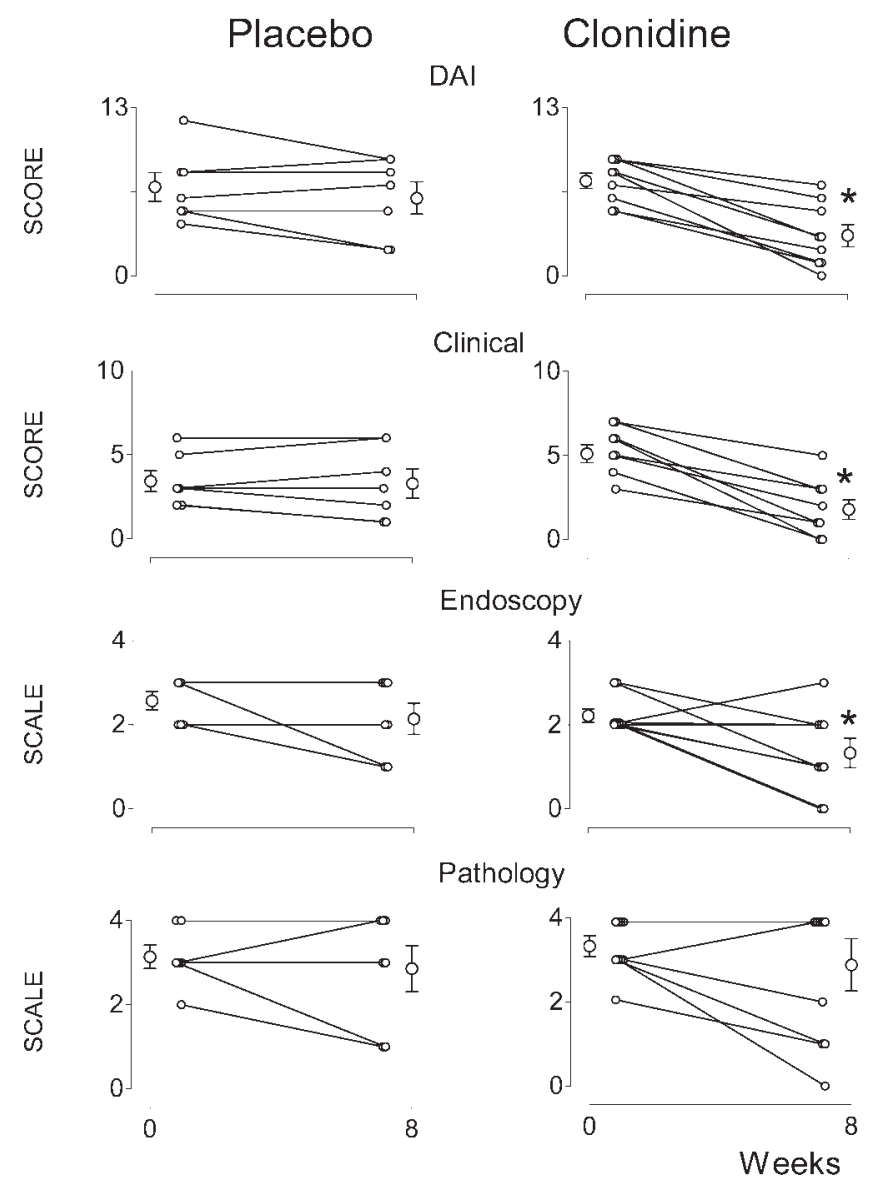

Fig. 6. Modifications in the severity of disease as assessed by the global Disease Activity Index (DAI) and clinical, endoscopic, and pathology scales after 8 wk of transdermal placebo and clonidine. Clinical scores and endoscopy scales are part of DAI (see Table 2). In the vertical axis, 0 indicates no activity of the disease and absence of symptoms or endoscopic and pathology abnormalities. Notice that clonidine administration was associated with a significant improvement of disease and amelioration of the endoscopic pattern. Pathology changes after 8 wk of drug therapy were less consistent. ${ }^{*} P<0.05$ vs. symptomatic phase of the disease (indicated by week 0 ). 
sympathetic efferent neurons that, in turn, may increase cytokines release $(10)$. Trophic changes $(7,21)$ and secondary alterations of blood flow regulation (19) might also sustain the vicious circle linking local gut inflammation and sympathetic activation (19).

It must be pointed out that a facilitating role played by the sympathetic nervous system in sustaining the local inflammatory mechanisms in experimental UC has been previously suggested by animal studies $(8,26)$. Indeed, sympathetic activity was found to promote local inflammatory mechanisms in $\mathrm{UC}$ after the onset of mucosal lesions (8). A reduced degree of colonic tissue damage was also observed in conscious rats with experimental colitis that had previously undergone sympathectomy compared with animals with an intact autonomic nervous system (26). However, it must be emphasized that other authors (18) described a reduced release of noradrenaline from myenteric plexus preparations of the inflamed distal colon and in noninflamed gut sites, suggesting a possible disruption, instead of an enhancement, of enteric sympathetic function in experimental UC.

The overall increase of sympathetic activity observed in UC patients of the present investigation is also in keeping with the data obtained by other studies performed in humans with active disease. In UC, the catecholamine content of rectal mucosa resulted in enhanced (31) and increased adrenergic innervation (21). Moreover, a relationship between autonomic hyperreflexia and duration and gravity of the disease has been observed in patients with UC on the basis of conventional autonomic tests, thus indirectly suggesting the existence of a hyperadrenergic condition (34).

Finally, it should be emphasized that patients with active UC in the present study showed reduced values of $\mathrm{HF}_{\mathrm{RR}}$, both in absolute and normalized units, suggesting a concomitant decrease of the vagal activity to the heart. This might reflect an overall diminished efficiency of the cholinergic anti-inflammatory pathway (39) to the gastrointestinal tract, a mechanism that might help maintain the bowel inflammatory state.

\section{Autonomic and Clinical Changes After Clonidine}

In nine subjects with acute UC, 8-wk sympathoplegic therapy resulted in a readjustment of the autonomic profile and in a concomitant clinical improvement of the disease. This finding further highlights the crucial role played by a sympathetic overactivity in sustaining the active phase of UC. Indeed, after clonidine, MSNA and plasma epinephrine decreased significantly. The LF-to-HF ratio and $\mathrm{LF}_{\mathrm{RR}}$ normalized units decreased, and the index of cardiac parasympathetic activity $\mathrm{HF}_{\mathrm{RR}}$ increased, suggesting a reduced sympathetic and enhanced parasympathetic modulation to the sinoatrial node.

As to the observed increase of parasympathetic modulation to the heart after clonidine, our results are in keeping with previous findings by Lazzeri et al. (22) in mild hypertensive patients and by Tank and colleagues obtained both in animals (37) and healthy humans (36). This latter investigation (36) indicates that $\alpha_{2}$-adrenoreceptor stimulation by clonidine increased baroreflex-mediated bradycardia during baroreceptor loading (36), a finding consistent with an increased cardiac parasympathetic tone.
Clonidine may shift the cardiac autonomic control toward a parasympathetic predominance by different mechanisms. Indeed, in animals, clonidine was found to reduce sympathetic outflow from the ventrolateral medulla neurons (16), thus leading to a relative parasympathetic predominance. In addition, it may stimulate $\alpha_{2}$-adrenoreceptors of the vagus dorsal motor nuclei (33), inducing direct central increase of cardiac parasympathetic modulation. Finally, peripheral stimulation of cardiac presynaptic $\alpha_{2}$-adrenoreceptors may mediate ACh release from cholinergic neurons of the heart (28) mimicking parasympathetic stimulation.

Plasma norepinephrine levels were only slightly reduced when UC improved. This finding might reflect the fact that sensitivity of plasma norepinephrine in sensing changes of the sympathetic activity may be limited, as previously observed in hypertensive patients (13).

The global clinical amelioration of the disease observed in the present study after clonidine is in accordance with the clinical and endoscopic findings observed by Lechin et al. (23), who had previously administered clonidine to patients with UC to mimic the beneficial effects of tioperazine on colonic motility.

Finally, a puzzling finding of the present work is the more pronounced reduction after clonidine of clinical symptoms and endoscopy abnormalities compared with the histological pattern. Indeed, after clonidine, histology was unchanged compared with the active phase of UC. However, permanent architectural abnormalities have been described to persist for months even in patients with clinically and endoscopically quiescent colitis (11). Therefore, the complete histological recovery might be achieved in the UC patients of the present work later than our 8-wk follow-up period.

\section{Study Limitations}

It must be pointed out that the sympathetic overactivity observed in UC patients of the present study and the reduced cardiac vagal modulation may not be a disease-specific feature of UC but rather a generic autonomic trait due to the inflammatory state. With respect to this issue, the design of the present study does not allow any conclusion because the autonomic profile of other types of colitis, such as infectious, uremic, or autoimmune diseases were not assessed. However, although not disease specific, the sympathetic overactivity observed in the active phase of UC seems to play a crucial role in generating and sustaining the bowel inflammatory state, given the fact that the administration of a sympathoplegic drug, such as clonidine, was associated with a clinical amelioration of the disease, whereas the placebo was ineffective.

As to the use of clonidine, the present investigation must not be considered a pharmacological trial. Instead, we focused on one of the potential pathophysiological mechanisms underlying the acute phase of UC. Given the finding of an abnormally increased sympathetic activity, we tried to reduce it by giving a centrally acting sympathoplegic drug, such as clonidine. Restoration of an autonomic profile, similar to that observed in healthy controls, was associated with a clinical and endoscopic improvement of the disease. 


\section{Perspectives}

In the present study, we reported findings supporting an important role of sympathetic activity in the pathophysiology of UC. Moreover, the association between a reduction of sympathetic overactivity by clonidine and the clinical improvement of the disease is striking and raises the possibility that a $\alpha_{2}$-adrenoreceptor agonist might be routinely added to a regular therapeutic regimen in $\mathrm{UC}$, thus possibly decreasing the need of a large dosage of other drugs, such as steroids, and increasing patient drug compliance. However, specifically designed clinical trials are needed to evaluate whether drug manipulation of autonomic profile might represent an additional therapy for UC.

In conclusion, our observations highlight the crucial role played by an enhanced sympathetic activity, possibly in maintaining the inflammatory process of the gut. In this context, clonidine administration was found to reduce the abnormally increased sympathetic activity and improve clinically active UC.

\section{REFERENCES}

1. Akselrod S, Gordon D, Ubel FA, Shannon DC, Barger AC, and Cohen RJ. Power spectrum analysis of heart rate fluctuation: a quantitative probe of beat-to-beat cardiovascular control. Science 213: $220-222,1981$.

2. Appenzeller $\mathbf{O}$ and Oribe $\mathbf{E}$. The enteric nervous system. In: The Autonomic Nervous System: an Introduction to Basic and Clinical Concepts. Amsterdam: Elsevier, 1997, p. 303-331.

3. Ardizzone $\mathbf{S}$ and Bianchi Porro G. A practical guide to the management of distal ulcerative colitis. Drugs 55: 519-542, 1998.

4. Aziz $\mathbf{Q}$ and Thompson DG. Brain-gut axis in health and disease. Gastroenterology 114: 559-578, 1998

5. Baselli G, Cerutti S, Civardi S, Liberati D, Lombardi F, Malliani A, and Pagani M. Spectral and cross-spectral analysis of heart rate and arterial blood pressure variability signals. Comput Biomed Res 19: 520 534, 1986.

6. Bishop AE and Polak JM. The gut and the autonomic nervous system. In: Autonomic Failure: a Textbook of Clinical Disorders of the Autonomic Nervous System, edited by Bannister R and Mathias CJ. Oxford: Oxford University Press, 1992, p. 160-177.

7. Collins SM. The immunomodulation of enteric neuromuscular function: implications for motility and inflammatory disorders. Gastroenterology 111: 1683-1699, 1996.

8. Collins SM, McHugh K, Jacobson K, Khan I, Riddel R, Murase K, and Weingarten HP. Previous inflammation alters the response of the rat colon to stress. Gastroenterology 111: 1509-1515, 1996.

9. Esler M, Jennings G, Lambert G, Meredith I, Horne M, and Eisenhofer G. Overflow of catecholamine neurotransmitters to the circulation: source, fate, and function. Physiol Rev 70: 963-985, 1990.

10. Felder RB, Francis J, Zhang Z, Wei S, Weiss RM, and Johnson AK. Heart failure and the brain: new perspective. Am J Physiol Regul Integr Comp Physiol 284: R259-R276, 2003.

11. Fenoglio-Preiser CM. Gastrointestinal Pathology: an Atlas and Text. Philadelphia: Lippincott-Raven, 1999, p. 672.

12. Fiocchi C. Inflammatory bowel disease: etiology and pathogenesis. Gastroenterology 115: 182-205, 1998.

13. Folkow B, Di Bona GP, Hjemgdahl P, Thoren PH, and Wallin BG. Measurements of plasma norepinephrine concentrations in human primary hypertension: a word of caution on their applicability for assessing neurogenic contributions. Hypertension 5: 399-403, 2000.

14. Furlan R, Guzzetti S, Crivellaro W, Dassi S, Tinelli M, Baselli G, Cerutti S, Lombardi F, Pagani M, and Malliani A. Continuous 24-h assessment of the neural regulation of systemic arterial pressure and RR variabilities in ambulant subjects. Circulation 81: 537-547, 1990.

15. Hahn MK. Norepinephrine transporter dysfunction. In: Primer on the Autonomic Nervous System, edited by Robertson D, Biaggioni I, Burnstock G, and Low PA. London: Elsevier, 2004, p. 280-282.
16. Head GA. Cardiovascular functions of central noradrenergic neurons in rabbits. Clin Exp Pharmacol Physiol 18: 51-54, 1991.

17. Jacob G, Shannon JR, Costa F, Furlan R, Biaggioni I, MosquedaGarcia R, Roberson RM, and Robertson D. Abnormal norepinephrine clearance and adrenergic receptor sensitivity in idiopathic orthostatic intolerance. Circulation 99: 1706-1712, 1999.

18. Jacobson K, McHugh K, and Collins SM. Experimental colitis alters myenteric nerve function at inflamed and noninflamed sites in the rat. Gastroenterology 109: 718-722, 1995.

19. Janig W. Pain and the sympathetic nervous system: pathophysiological mechanisms. In: Autonomic Failure: a Textbook of Clinical Disorders of the Autonomic Nervous System, edited by Bannister R and Mathias CJ. Oxford, UK: Oxford University Press, 1992, p. 231-251.

20. Jasson S, Medigue C, Maison-Blanche P, Montano N, Meyer L, Vermeiren C, Mansier P, Coumel P, and Malliani A. Instant power spectrum analysis of heart rate variability during orthostatic tilt using a time/frequency-domain method. Circulation 96: 3521-3526, 1997.

21. Kyosola K, Penttila O, and Salaspuro M. Rectal mucosal adrenergic innervation and enterocromaffin cells in ulcerative colitis and irritable colon. Scand J Gastroenterol 12: 363-367, 1977.

22. Lazzeri C, La Villa G, Mannelli M, Janni L, Barletta G, Montano N, and Franchi F. Effects of clonidine on power spectral analysis of heart rate variability in mild essential hypertension. J Auton Nerv Syst 74: $152-159,1998$.

23. Lechin F, van der Dijs B, Insausti CL, Gòmez F, Villa S, Lechin AE, Arocha L, and Oramas $\mathbf{O}$. Treatment of ulcerative colitis with clonidine. J Clin Pharmacol 25: 219-226, 1985.

24. Malliani A. Cardiovascular sympathetic afferent fibers. Rev Physiol Biochem Pharmacol 94: 11-74, 1982.

25. Malliani A, Pagani M, Lombardi F, and Cerutti S. Cardiovascular neural regulation explored in the frequency domain. Circulation 84: 482-492, 1991.

26. McCafferty DM, Wallace JL, and Sharkey KA. Effects of chemical sympathectomy and sensory nerve ablation on experimental colitis in the rat. Am J Physiol Gastrointest Liver Physiol 272: G272-G280, 1997.

27. Nicogossia AE, Huntoon CL, and Pool SL. Cardiopulmonary function. In: Space Physiology and Medicine, Philadelphia: Lea \& Fabiger, 1994, p. 286-304.

28. Nosaka S, Murata K, Kobayashi MCZB, and Maruyama J. Inhibition of baroreflex vagal bradycardia by activation of the rostral ventrolateral medulla in rats. Am J Physiol Heart Circ Physiol 279: H1239-H1247, 2000.

29. Pagani M, Lombardi F, Guzzetti S, Rimoldi O, Furlan R, Pizzinelli P, Sandrone G, Malfatto G, Dell'Orto S, Piccaluga E, Turiel M, Baselli G, Cerutti S, and Malliani A. Power spectral analysis of heart rate and arterial pressure variabilities as a marker of sympathovagal interaction in man and conscious dog. Circ Res 59: 178-193, 1986.

30. Pagani M, Montano N, Porta A, Malliani A, Abboud F, Birkett C, and Somers VK. Relationship between spectral components of cardiovascular variabilities and direct measures of muscle sympathetic nerve activity in humans. Circulation 95: 1441-1448, 1997.

31. Penttila O. Studies on rectal mucosal catecholamines in ulcerative colitis. Ann Clin Res 7: 32, 1975.

32. Pullan RD, Rhodes J, Ganesh S, Mani V, Morris JS, Williams GT, Newcombe RG, Russell MAH, Feyerabend C, Thomas GAO, and Sawe U. Transdermal nicotine for active ulcerative colitis. $N$ Engl J Med 330: 811-815, 1994.

33. Robertson HA and Leslie RA. Noradrenergic $\alpha-2$ binding sites in vagal dorsal motor nucleus and nucleus tractus solitarius: autoradiographic localization. Can J Physiol Pharmacol 63: 1190-1194, 1985.

34. Straub RH, Antoniou E, Zeuner M, Gross V, Scholmerich J, and Andus T. Association of autonomic nervous hyperreflexia and systemic inflammation in patients with Crohn's disease and ulcerative colitis. J Neuroimmunol 80: 149-157, 1997.

35. Sutherland LR, Martin F, Greer S, Robinson M, Greenberger N, Saibil F, Martin T, Sparr J, Prokipchuk E, and Borgen L. 5-Aminosalycil acid enema in the treatment of distal ulcerative colitis, proctosigmoiditis and proctitis. Gastroenterology 92: 1894-1898, 1987.

36. Tank J, Diedrich A, Szczech E, Luft FC, and Jordan J. $\alpha$-2 Adrenergic transmission and human baroreflex regulation. Hypertension 43: 10351041, 2004. 
37. Tank J, Jordan J, Diedrich A, Obst M, Plehm R, Luft FC, and Gross V. Clonidine improves spontaneous baroreflex sensitivity in conscious mice through parasympathetic activation. Hypertension 43: 1042-1047, 2004.

38. Task Force of the European Society of Cardiology, and the North American Society of Pacing and Electrophysiology. Heart rate variability: standards of measurements, physiological interpretation, and clinical use. Circulation 93: 1043-1065, 1996.

39. Tracey KJ. The inflammatory reflex. Nature 420: 853-859, 2002.

40. Truelove SC and Richards WCD. Biopsy studies in ulcerative colitis. $\mathrm{Br}$ Med J June 9: 1315-1321, 1956.
41. Vallbo AB, Hagbarth KE, Torebjork HE, and Wallin BG. Somatosensory, proprioceptive and sympathetic activity from peroneal nerves. Physiol Rev 59: 919-957, 1979.

42. Whitehead WE. Effects of psychological factors on gastrointestinal function. In: Pathogenesis of Functional Bowel Disease, edited by Snape W. New York: Plenum, 1989, p. 37-53.

43. Wingate DL. Autonomic dysfunction and the gut. In: Autonomic Failure: a Textbook of Clinical Disorders of the Autonomic Nervous System, edited by Bannister R and Mathias CJ. Oxford, UK: Oxford University Press, 1992, p. $510-528$.

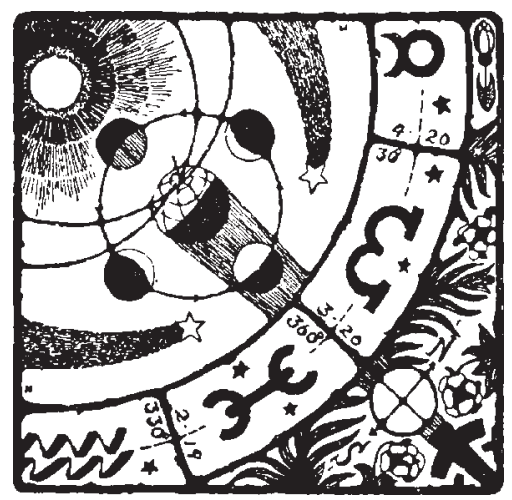

